\title{
Non-linear forecasting of the state of a socio-eco-oriented innovative economy in the conditions of systemic crises
}

\author{
Sultan Ramazanov ${ }^{1, *}$, Oleksandr Chernyak $^{2}$, Bogdan Tishkov $^{1}$, Renat Ahmedov ${ }^{1}$, and Oleksandr Honcharenko ${ }^{1}$ \\ ${ }^{1}$ Kyiv National Economic University named after Vadym Hetman, Economics Information Systems Department, Kyiv, Ukraine \\ ${ }^{2}$ Taras Shevchenko National University of Kyiv, Department of economic cybernetics, Kyiv, Ukraine
}

\begin{abstract}
The paper deals with the problem of sustainable development and innovative integral modeling and forecasting approach in the management of technogenic objects and processes (TOP) as a system of socio-eco-economic and humanitarian type (SEEH). Based on the use of information and innovation technologies in order to forecast the non-linear dynamics of eco-economic and socio-humanitarian systems, integrated stochastic models of objects and processes were developed and studied, suitable for the conditions of systemic crises. The paper handles the aspect of integration of 4 business and functioning areas of the modern complex systems. It proposes a general conceptual integrated model, generalized synergetic model of dynamics, considering different uncertainty (stochastic and chaotic components). The paper examines the aspects of integration of multiple business areas and sectors of the modern complex systems functioning and developing under the present conditions of non-linearity, instability and crises. An integrated stochastic non-linear phase-space growth dynamics model was developed and studied to forecast the development of the state of an innovative economy. The paper looks into the aspects of activity management of the modern complex systems functioning and developing under the present conditions of instability.
\end{abstract}

\section{Introduction. Problem analysis and statement}

The global goal of this research is to create a general conception, principles, methodology, methods, models and information technologies and systems for making managerial decisions for sustainable, safe, secure and viable development of economy, environmental economy and socio-humanitarian systems under the conditions of uncertainty, risks, threats and crises [1-8].

The knowledge of the scientific potential on economics was assigned to V. Heyets [9], T. Klebanova [10], I. Lyashenko [11] and others. Methodology of regional scientific and technological progress (STP) in aggregative models in economics was described by S. Aywazyan, M. Afanasyev, V. Rudenko [12], G. Kleiner [13]. The problems of mathematical modeling of complex economical systems of ecology and economy were researched by a lot of academics, such as D. Ford, V. Leontyev [14], J. Tsukui, J. Murakam [15], V. Brock [16], O. Tahvonen, J. Kuuluvainen [17]. The most comprehensive investigation was fulfilled by Ya. Vagapova [18], which is a description of the national economy with a system of nonlinear differential equations, based on the A. Moiseyev model [19].

The conception of sustainable, safe, secure and viable development provides for a change in the paradigms of the traditional economy, humanization and ecologization of its core principles, search for general approaches and coordination between the development conceptions of environmental and economic systems.
The problem of sustainable development features the following aspects: systemic, synergetic, environmental, economic, mathematical, informational, philosophical, legal, educational, etc. The paper handles the aspect of integration of 4 business and functioning areas of the systems: socio-eco-economic and humanitarian, including spiritual-moral and cultural technologies. The sustainable development paradigm requires transition from the investigation of separate functioning isolated systems to the investigation of integral evolving systems, actualization and strengthening of system-synergetic links of an open system and its environment. The existing "monodisciplinary" conceptions, linear static models, reproductive-representative methods (reflecting the linearity of our knowledge), profitability and optimality criteria should give place to interdisciplinary, system-synergetic, evolutionary non-linear models and methods of forecasting and knowledge acquisition, management (reflecting the non-linearity and nonGaussian character of the laws of nature and society), criteria of rationality, local optimality, and correspondence to the "common sense". "Interdisciplinary" paradigm is understood not in the intuitive, technocratic relationship of disciplines, accumulation of quanta of knowledge and skills, but in the actualization of individual and public intellect, education and learning of new self-organized and sustainable structures [19-25].

Under the current complex conditions and as the modern economy advances, a need arises to consider its forecasting problems in a new fashion. This is connected

"Corresponding author: sramazanov@i.ua 
with the increased influence of globalization processes and uncertainty and risk factors on the economy and business performance of individual countries and regions. Existing forecasting methods and models rely, to a considerable extent, on the application of econometric models. This paper analyzes the opportunities of development forecasting on the basis of the approximation of the integral stochastic growth model in the form of recurrent equations formed taking into account the increment properties of the Wiener random processes. This considers only current or initial state of the economy.

Only integration of the modeling methods for socioeconomic, environmental, cultural and spiritual and other processes will ensure sustainability and viability of development of the entire system [1-5].

Also, note that a system-thinking and active person usually forecasts and considers his/her performance results, compares his/her capabilities, considers environmental interests, those of future generations and the need for noospheric development.

The purpose of the paper is to develop and investigate problems of non-linear forecasting, in particular on the basis of the integral socio-ecoeconomic stochastic model of the dynamics of technogenic objects and processes functioning under the conditions of systemic crises.

The problem of modeling, forecasting, optimal management and decision making in socio-ecologicaleconomic systems and in science as a whole is the main and urgent. The purpose of this work is to study the methods, principles and models for forecasting and management used in various socioeconomic processes and systems, as well as the introduction of a new class of models for solving problems of modeling and prediction of socio-ecological and economic processes. The work is based on the analysis of modern methods of dynamic modeling and forecasting of various processes and systems [1-5].

Description of key findings. In this paper, technogenic objects and processes (TOP) mean technogenic production enterprises (TPE) or technogenic regional production operations (TRPO), or technogenic regions (TR), technogenic economic object (TEO), etc. Let us designate all these as TOP.

Under the current conditions of instability and crises, the problem of developing analysis, modeling, forecasting and decision-making methods and technologies for sustainable development of viable socio-economic systems is becoming even more topical. These systems are characterized by the complexity of the structure and behavior, synergeticity, non-linearity and have other "NO" and "MANY" factor characteristics.

Moreover, another very important problem includes investigation of systems with integral properties, i.e. systems including in their structure socio-economic and humanitarian subsystems (SEEH) as the systems of the future (noospheric type systems). Local production systems of regional level, such as technogenic industrial systems (TIS) are also SEEH type systems.

To investigate and solve these problems, management and decision-making methods and models based on the object-oriented approach have been traditionally used. However, a series of scientific publications and scientific schools have recently appeared, which lay emphasis on the importance and necessity of recording the behavior dynamics of a management subject, use of the subject-oriented approach and reflection to resolve the said tasks [1-5].

The paper proposes the review and analysis of some findings obtained in the recent years by different authors for macro- and micro-modeling of the dynamics of ecoeconomic and socio-humanitarian systems and processes which are attributed to TOP and function and develop under the complex conditions of non-linearity, instability and crises.

Most of the previously created models of socio-ecoeconomic systems (SEES) have theoretical and deterministic nature and are rather problematic from the viewpoint of the adequacy and availability of information for their implementation. In this connection, the objective of technogenic regional production operations (TRO) management under the conditions of crisis causes the objective necessity of improving methods, models and information technologies on the basis of stochastic equations for SEEH management.

The key requirement of the sustainable development paradigm includes creation of existence conditions for future generations by means of restricted use of natural resources, establishment of natural resources and environment recovery cycles together with social capital development - all these can only be realized based on the use of scientific and technical achievements on the back of the high innovation activity. Therefore, the innovative development paradigm conceptually justifies the way for achievement of sustainable growth of TRO (TOP) with the help of development of the human potential and reduction of the TRO load on people and the environment.

To improve the TRO management efficiency amid crisis, one should positively influence all its structural components on which the success of the long-term TRO activities depends. Sustainable development of the TRO requires such choice and combination of its component parts to ensure harmonious functioning of the TRO as a whole. One of the main drivers of increasing the operating efficiency of the TRO includes intensification of production, which is crucially influenced by science. Intellectual capital of the TRO is an internal resource capable of giving new innovative advantages to it. It is important that the innovation activities of the TRO aimed at getting over crisis events result in the increased level of their systematicity, and a synergetic effect was obtained owing to this.

The papers [1-5] studied the process of improvement of the technogenic regional production operations management mechanism through development of methods, models and information technologies of socioeco-economic management (SEEM) amid crisis. The mathematical and conceptual models were proposed, and scenario calculations were carried out for the TRO management simulation model.

This research represents the development of the findings of the authors' papers [1-5] for eco-economic 
modeling and management in case of recording of stochastic impact factors, and we hope that the presented material will be useful for both prominent researchers in this area of science and young scientists.

\section{Main results}

The conceptual model of forecasting and management of eco-economic processes (EEP) of a technogenic economic object (TEO) under the conditions of "NO and MANY factors" available, can be presented in the form of a set-theoretical model as a tuple $[3,5]$ :

$$
\left\langle X, Y, F, H, R, E, \Omega, T, G, K_{u}, K_{p}, P, U\right\rangle,
$$

where $X$ - set of possible states of the technogenic economic object; $Y=\left\langle Y^{\mathrm{ekn}}, Y^{e k l}\right\rangle-$ total output of the technogenic economic object, where $Y^{\text {ekn }}$ - productive set (i.e. "useful output"), and $Y^{e k l}-$ set of pollutions (i.e. "harmful output"); $F=\left\langle F^{\mathrm{ekn}}, F^{e k l}\right\rangle$ - model reflection of the TEO; $H=\left\langle H^{e k n}, H^{e k l}\right\rangle$ - general operator of observations (measurements); $R$ - resource set (i.e. main controlled entry of the TEO); $E$ - set of unidentified factors (both external and internal, i.e. both additive and multiplicative), in particular this is a set of stochastic, fuzzy, multiple or mixed uncertainty; $\Omega$ - set of constraints; $T=\left[t_{0} ; t_{k}\right]$ - time interval of the TEO functioning and development; $G$ - target set; $K_{u}-$ generalized eco-economic control criterion (EECC); $K_{p}-$ generalized forecasting optimization criterion (FOC); $P$

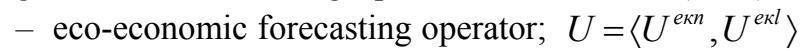

- eco-economic control (EEC) vector. Designations "eкn" and "eкl" correspond to economic and environmental variables.

Then the task of optimal eco-economic forecasting, i.e. determining a predictor for both internal and external processes can be formulated as follows: determine the estimate $\hat{x}\left(t_{k} T+\Delta\right), \Delta=\Delta_{0}, \Delta_{1}, \ldots$ of the state vector $x\left(t_{k}+\Delta\right)$ with the preset forecast step $\Delta$ on the basis of multiple eco-economic observations $\left\{y(t), t \in\left[t_{0}, t_{k}\right]\right\}$

and based on the preset FOC $K_{p}$.

Now the EEC task is to determine the effective integral management vector $U=\left\langle U^{\text {ekn }}, U^{\text {exl }}\right\rangle$ on the bases of the estimates $\hat{x}\left(t_{k} T+\Delta\right), \Delta=\Delta_{0}, \Delta_{1}, \ldots$ and nonlinear dynamic eco-economic model of the TEO that ensures achievement of the goal $G$ with a preset generalized eco-economic criterion $K_{u}$ and constraints $\Omega$ taking into account the uncertainty and risks.

The multiplicative-additive stochastic model with the chaotic dynamics can be generally presented as vector equations:

$$
\begin{aligned}
& \dot{x}=A(t) x(t)\left[X^{0}-x(t)\right]+D(t), \\
& A(t)=a(t) \lambda(t) \zeta(t), D(t)=d(t) \xi(t)
\end{aligned}
$$

or, multiplicative-additive stochastic model with the chaotic dynamics and with management, i.e. taking into account action managers:

$$
\begin{aligned}
& \dot{x}=A(t) x(t)\left[X^{0}-x(t)\right]+D(t)+P(t), \\
& A(t)=a(t) \lambda(t) \zeta(t), \quad D(t)=d(t) \xi(t), \\
& P(t)=p(t) \psi(t) u(t) .
\end{aligned}
$$

The observation model is represented as follows:

$$
y(t)=H(t) x(t)+\eta(t) .
$$

Here, the following designations are used: $\xi(t), \zeta(t)$, $\eta(t)$ - multiplicative-additive stochastic components in the models (2)-(4), and $\lambda(t)$ - chaotic component in the system model (2). Other designations are shown below.

The integral socio-eco-economic dynamic behavioral model with spiritual and moral variables can be conceptually presented in a general (block) form $[4,5]$ :

$$
\left\{\begin{array}{l}
\dot{X}_{1}=f_{1}\left(X_{1}, X_{2}, X_{3}, X_{4} ; P_{1}, \xi_{1}\right), \\
\dot{X}_{2}=f_{2}\left(X_{1}, X_{2}, X_{3}, X_{4} ; P_{2}, \xi_{2}\right), \\
\dot{X}_{3}=f_{3}\left(X_{1}, X_{2}, X_{3}, X_{4} ; P_{3}, \xi_{3}\right), \\
\dot{X}_{4}=f_{4}\left(X_{1}, X_{2}, X_{3}, X_{4} ; P_{4}, \xi_{4}\right),
\end{array}\right.
$$

where $X=\left(X_{1}, X_{2}, X_{3}, X_{4}\right)$ - combined vector of behavioral variables and states of the socio-ecoeconomic system taking into account the humanitarian level variable $(\mathrm{SEEH})-X_{4}$, as well as in (5) $X_{1}=X_{1}(t)-$ vector of economic variables; $X_{2}=X_{2}(t)$ - vector of environmental variables (pollution variables); $X_{3}=X_{3}(t)-$ vector of social variables; $P=\left(P_{1}, P_{2}, P_{3}, P_{4}\right)-$ cumulative vector of SEES parameters (intra-system and environmental); $\Xi=\left(\xi_{1}, \xi_{2}, \xi_{3}, \xi_{4}\right)$ - vector of external random and unidentified variables. For example, for technogenic production systems (TPS) [3] $X_{1}=\left(K_{1}, L_{1}, I\right.$, $\tau, C), C=\left(C_{1}, C_{2}, C_{3}, C_{4}\right)-$ vector of some consumption (expenditure) parameters, and $C_{1}-$ value of social consumption (i.e. for wages, etc.); $C_{2}=C_{e}-$ consumption for the environment; $C_{3}=C_{s}$ - consumption for safety and security; $C_{4}=C_{i}-$ investment volume for innovation, information and humanitarian technologies.

Synergetic dynamics model of the non-linear stochastic system with the chaotic behavior:

$$
\begin{aligned}
& \dot{x}_{i}=\lambda_{i} \xi_{i}(t) x_{i}(t)\left[\Sigma_{X} \pm \sum_{j=1}^{n} a_{i j}(t) \prod_{k=1}^{j} x_{k}(t)\right]+ \\
& +\sum_{l=1}^{3} d_{i l} \frac{\partial^{2} x_{i}}{\partial r_{l}^{2}}+w_{i}+b_{i} u_{i}(t), \\
& i=\overline{1, n}, \overline{x_{i}}(0)=x_{i 0},
\end{aligned}
$$

where $\left\langle\xi_{i}, w_{i}\right\rangle$ - stochastic components of the model; $\left\{a_{i j}(t)\right\}$ - non-stationary components of the model; $\left\{d_{i l}\right\}$ - diffusion coefficients determining the level of distribution of the state variables; $\Sigma_{X}-$ aggregate maximum (maximum allowable) value of the vector $\boldsymbol{x}$, $\left\{\lambda_{i}\right\}-$ set of parameters causing chaotic nature. 
In particular, this model can be also represented as an equation system:

$$
\begin{gathered}
\partial X_{i} / \partial t= \\
=A_{i}\left[\xi _ { i } \left(r_{i} X_{i}-\right.\right. \\
\left.\left.-\sum_{j \neq i} b_{i j} X_{i} X_{j}-a_{i} X_{i}^{2}\right)+D_{i}(x, y) \Delta X_{i}\right]+\varsigma_{i}+u_{i},
\end{gathered}
$$

where $X_{i}$ - coordinates of the system state vector, where $X_{i} \equiv X_{i}(t, x, y) ; i, j=1,2, \ldots, n$ - reproduction (duplication, growth, development, etc.) coefficient; $a_{i}-$ saturation parameter limiting growth (reproduction); $b_{i j}-$ subsystem interaction parameter (between business entities); $D_{i}(x, y)$-diffusion factor of the $i$-th subsystem (of the economic entity) in the point $(x, y) ; \xi_{i} \equiv \xi_{i}(t, x, y)$ and $\zeta_{i} \equiv \zeta_{i}(t, x, y)$ - stochastic multiplicative and additive components of the model, respectively; $u_{i} \equiv u_{i}(t, x, y)-$ coordinates of the management vector, i.e. vector of managerial decisions; $A_{i}$ - scaling factor $\nabla$ - Laplacian, i.e. $\nabla(*)=\partial^{2}(*) / \partial x^{2}+\partial^{2}(*) / \partial y^{2}$ and $t \in[0, T]-$ time interval of the system functioning and development.

Such models describe and cover rather wide class of complex processes and systems, including the noospheric model of stable development (NMSD) [5].

The system has synergetic (or cybernetic) description, if the effectively built operator $D$ is such that the state of the system at each point of time $t \in\left(t_{0}, T\left(t_{0}\right)\right)$ can be built based on the vector values $x(\tau), \tau \in\left(t_{1}, t_{0}\right)$, provided that all the external managing actions are fixed:

$$
x(t)=D(x(\tau), \varepsilon, \eta, u), t \in\left(t_{0}, T\left(t_{0}\right)\right), \tau \in\left(t_{1}, t_{0}\right),
$$

where $\varepsilon(t, r)$ - random action with the known probabilistic characteristics; $\eta(t, r) \in G_{\eta}-$ action predetermined by the extent of uncertainty; $G_{\eta}, u \in R^{k}$ - managing actions; $r$ - spatial variable (vector).

Consequently, the integrated stochastic model of the growth dynamics for forecasting the development of the innovative economy can be represented in the form of the following system of difference equations:

$$
\begin{gathered}
K_{t+\Delta}=K_{t}-\left(\alpha K_{t}-I_{t}+C_{t}+D_{t}+B_{t}\right) \Delta+\sigma_{K} \Delta \xi_{t},(9) \\
Y_{t}=F\left(K_{t}, L_{t}, R_{t}, \xi_{t}\right)= \\
=\left[\beta_{1} K_{t}^{\frac{\delta-1}{\delta}}+\beta_{2} L_{t}^{\frac{\delta-1}{\delta}}+\beta_{3} R_{t}^{\frac{\delta-1}{\delta}}\right]^{\frac{\delta}{\delta-1}}+\sigma_{Y} \xi_{t}, \\
\text { or } \left.\quad Y_{t}=A \tau_{t} \mid\left(1-\alpha_{K}^{1}(t)-\alpha_{K}^{2}(t)-\alpha_{K}^{3}(t)\right) K_{t}\right]^{\alpha_{1}} \\
\quad \cdot\left[\left(1-\alpha_{L_{2}}^{1}(t)-\alpha_{L_{2}}^{2}(t)\right) L_{2}\right]^{\alpha_{2}}+\sigma_{Y} \xi_{t}, \\
\quad L_{t+\Delta}=L_{t}+\left(\gamma L_{t}-\gamma_{Z} Z_{t}+\gamma_{C} C_{t}\right) \Delta+ \\
\quad+\sigma_{L} \Delta \xi_{t}, L(0)=L_{0}, \\
R_{t+\Delta}=R_{t}+\left(\gamma_{R} R_{t}+\gamma_{K} K_{t}-Y_{t}-\gamma_{L} L_{t}\right) \Delta+ \\
+\sigma_{R} \Delta \xi_{t}, R(0)=R_{0}
\end{gathered}
$$

or

$$
\begin{aligned}
& R_{t+\Delta}=R_{t}+\left(d\left(K_{t}, L_{t}, R_{t}\right)+\gamma_{K} K_{t}-\gamma_{L} L_{t}-Y_{t}\right) \Delta+ \\
& +\sigma_{R} \Delta \xi_{t} \text {, } \\
& Z_{t+\Delta}=Z_{t}+\left(g\left(Z_{t}\right)+f^{*}\left(C_{t}, K_{t}, L_{t}, R_{t}\right)\left(1-\eta C_{t}\right)\right) \Delta+ \\
& +\sigma_{Z} \Delta \xi_{t}, Z(0)=Z_{0} \text {, } \\
& z_{t+\Delta}=z_{t}+\left(\delta_{z} z_{t}+E\left[\phi_{t}\right]^{v_{1}} \cdot\left[\alpha_{L}^{1}(t) L_{t}\right]^{v_{2}} .\right. \\
& \left.\cdot\left[\alpha_{K}^{2}(t) K_{t}\right]^{v_{3}}\right) \Delta+\sigma_{z} \Delta \xi_{t} \text {, } \\
& \phi_{t+\Delta}=\phi_{t}+\left(\delta_{\phi} \phi_{t}+G\left[\phi_{t}\right]^{\gamma_{1}} \cdot\left[\alpha_{L_{1}}^{1}(t) L_{1_{t}}\right]^{\gamma_{2}} .\right. \\
& \left.\cdot\left[\alpha_{K}^{1}(t) K_{t}\right]^{\gamma_{3}} \cdot[s(t)]^{\gamma_{4}}\right) \Delta+\sigma_{\phi} \Delta \xi_{t} \text {, } \\
& L_{t+\Delta}=L_{1 t}+\left(\delta_{L_{1}} L_{1 t}+D\left[\phi_{t}\right]^{\eta_{1}} .\right. \\
& \left.\cdot\left[\alpha_{L_{1}}^{2}(t) L_{1 t}\right]^{\eta}{ }^{2}\right) \Delta+\sigma_{L_{1}} \Delta \xi_{t}, \\
& s_{t+\Delta}=s_{t}+\left(\delta_{s} s_{t}+H\left[\phi_{t}\right]^{v_{1}} \cdot\left[\varepsilon_{L}^{1}(t) L_{t}\right]^{v_{2}} .\right. \\
& \left.\cdot\left[\varepsilon_{K}^{2}(t) K_{t}\right]^{v_{3}} \cdot\left[\varepsilon_{z}^{3}(t) z_{t}\right]^{v_{4}}\right) \Delta+\sigma_{s} \Delta \xi_{t}, \\
& \tau_{t+\Delta}=\tau_{t}+\left\{\delta_{\tau} \tau_{t}+Q\left[\frac{\phi_{t+\Delta}-\phi_{t}}{\Delta}+\delta_{\phi} \phi_{t}\right]^{\beta_{1}} .\right. \\
& \cdot\left[\frac{L_{1 t+\Delta}-L_{1 t}}{\Delta}+\delta_{L_{1}} L_{1 t}\right]^{\beta_{2}}\left[\frac{s_{t+\Delta}-s_{t}}{\Delta}+\delta_{s} s_{t}\right]^{\beta_{3}} \text {. } \\
& \left.\cdot\left[\frac{z_{t+\Delta}-z_{t}}{\Delta}+\delta_{z} z_{t}\right]^{\beta_{4}}\right\} \Delta+\sigma_{\tau} \Delta \xi_{t} \text {, } \\
& K_{t+\Delta}=K_{t}-\left(\alpha K(t)+I_{h t}-C_{t}-D_{t}-B_{t}\right) \Delta+ \\
& +\sigma_{K} \Delta \xi_{t}, K(0)=K_{0} \text {, } \\
& I_{h t+\Delta}=I_{h t}-\left(r I_{h}(t)+h_{0} I_{0}\right) \Delta+ \\
& +\sigma_{I_{h}} \Delta \xi_{t}, I_{h}\left(t_{0}\right)=h_{0} I\left(t_{0}\right) \text {, } \\
& C_{t+\Delta}=C_{t}+\left\{A_{t} K_{t}-(1+\delta) K_{t}\right\} \Delta+A_{t} K_{t} \sigma_{C} \Delta \xi_{t},
\end{aligned}
$$

where $\Delta-$ step of the time interval (forecast step), for which calculations will be made; $\xi_{t}$ - realization of the random value which is subject to the standard normal law with the average equal to zero and dispersion equal to one. Also note that here $z(t), s(t), \tau(t), \varphi(t)$ are the indexes of the respective basic indicators.

Note that computer realization of the stochastic difference (discrete) equations (9) - (20) will require transition to the averaged variables $x(t)=(Y(t), K(t), L(t)$, $\left.R(t), Z(t), z(t), s(t), \tau(t), \varphi(t), I_{h}(t), C(t)\right)$, i.e. to their expectation values.

Optimal control / management criteria options. Ecoeconomic development strategy selection criterion: 


$$
\mathrm{M}[\Phi(C, Y, D, B)] \rightarrow \max ,
$$

where $\Phi(C, Y, D, B)$ - welfare function, and $M-$ expectation value symbol.

Consequently, the hierarchical optimization model of the system is obtained in the following form:

$$
\left\{\begin{array}{l}
\mathrm{M}\{Z\} \rightarrow \min , \mathrm{M}\{K\} \rightarrow \max , \\
\dot{Z}=\chi(Z, K, L, R, c), \dot{K}=\phi(K, L, R, c) .
\end{array}\right.
$$

In case of a "small" integral management model, utility function (UF) is a function of the parameters / variables $\tilde{u}\left(\alpha_{1}, \alpha_{2}, \alpha_{3}, \alpha_{4}\right)$, where $\left\{\alpha_{k}(t), k=1, \ldots, 4\right\}-$ shares of costs for non-manufacturing, environmental costs, R\&D efforts, for safety and security, innovation and information technologies, etc., and then the following relationship will be the optimality criterion:

$$
\Lambda(c, k, z, L, \tau, S)=\int_{t_{0}}^{T} \widetilde{u}\left(\alpha_{1}, \alpha_{2}, \alpha_{3}, \alpha_{4}\right) \exp (-\theta t) d t \rightarrow \max _{\left\{\alpha_{i}\right\} \in \widetilde{\Omega}} .
$$

To resolve the EEC tasks on the basis of the presented stochastic and deterministic models, one can use the known classical methods of optimal control with restrictions [3].

Generally, the optimization criterion can be represented as

$$
\Lambda(\vec{a})=\int_{t_{0}}^{T} \tilde{u}\left(\alpha_{1}, \alpha_{2}, \alpha_{3}, \alpha_{4}, \ldots\right) \exp (-\theta t) d t \rightarrow \max _{\left\{\alpha_{i}\right\} \in \widetilde{\Omega}} .
$$

This requires even higher integration of models and criteria (and restrictions) of management optimization and DP.

It is also important to consider criteria of random (stochastic) factors of multiplicative and adaptive impact in the models.

In other words,

$$
\Lambda(\vec{a})=M\left[\int_{t_{0}}^{T} \tilde{u}\left(\alpha_{1}, \alpha_{2}, \alpha_{3}, \alpha_{4}, \ldots\right) \exp (-\theta t) d t\right] \rightarrow \max _{\left\{\alpha_{i}\right\} \in \Omega} .
$$

As a criterion in tasks similar to the one in question, objective functional of maximization of the average per capita consumption of employee within the temporary interval is usually assumed $[0, T]$ :

$$
\int_{0}^{T} \frac{C(t)}{L_{1}(t)+L_{2}(t)} \exp (-\theta t) d t \rightarrow \max
$$

where $\theta>0$ - discount factor that reflects the extent of giving preference to the future consumption over the current one.

Note that the criterion (24) is only a private (economic) criterion. Therefore, socio-ecological, innovation and other optimality criteria are also required integrally!

Control synthesis task in stochastic systems. Let us consider the optimal management synthesis task in stochastic linear systems [6].
Phase state of the system means the vector $x=\left(x_{1}, x_{2}\right.$, $\ldots, x_{n}$ ), where $x_{i}, i=1, \ldots, n$-aggregate of data describing the $i$-th sector of the system. Let us assume that all the sectors are provided with some management levers: $u=\left(u_{1}, u_{2}, \ldots, u_{n}\right)$.

Each of the listed parameters, in its turn, represents a vector composed of the numeric values of different characteristics of the system dynamics. Let us assume that $\left[t_{0}, t_{k}\right]$ - reviewed system development period. Let us designate as $\xi$ the vector of some additional parameters determined by means of forecasting using statistical data of the system, or which are constants.

Consequently, the dependence is studied of each variable $x_{i}, i=1, \ldots, n$ from the managing variables $u_{i}$ of the system states and the vector of parameters $\xi$.

The linearized system model looks as follows:

$$
\begin{gathered}
\dot{x}(\xi, t)=A(\xi) x(\xi, t)+B(\xi) u(\xi, t), \\
x(\xi, t) \in R^{n}, u(\xi, t) \in R^{m}, \\
A(\xi) \in R^{n \times m}, B(\xi) \in R^{n \times m} ;
\end{gathered}
$$

where $A(\xi)=\left|A_{i j}(\xi)\right|, \quad B(\xi)=\left|B_{i j}(\xi)\right|-$ matrices with parametric uncertainty; $x(\xi, t)=\left[x_{i}(\xi, t)\right]-$ system state vector; $u(\xi, t)=\left[u_{i}(\xi, t)\right]-$ management vector; $\xi$ - vector of stochastic processes.

The task of optimal management synthesis for a stochastic dynamic system is posed as follows: to find an optimal management law in the form of [6]:

$$
u(\xi, t)=-L \hat{x}(\xi, t)
$$

and the objective functional for optimization (minimization) should be predetermined in the following form:

$$
J=\int_{\Omega}\left\{\int_{0}^{t_{k}}\left(x^{T}(\xi, t) \Theta x(\xi, t)+u^{T}(\xi, t) R u(\xi, t)\right) d t\right\} \rho(\xi) d \xi .
$$

In a simple case, linear observation equation may be used in the following form: $y(t)=H(t) x(t)+\eta(t)$. In this equation, the observation $H(t)$ is represented by a stochastic observation matrix, which can be preset in practical terms, and $\eta(t)$ is the "white noise" type process, which can be preset based on statistical data. In doing so, two independent tasks are to be resolved based on the separation principle [6].

In the early papers of the first author of this paper, the task of optimal evaluation (filtration) under the conditions of multiplicative-additive mixtures was resolved using the integral description of the filter [6].

However, the filtration task can also be conveniently resolved using the Kalman filter for the linear case and the Stratonovich filter for the non-linear right part of the dynamics equation [6]. State estimates are found as the conventional average values with the building and solution of the non-linear dispersion equations of the Riccati type. The filter gives a solution for the stochastic differential equation. It can be implemented in the form of the known closed loop system for the distribution density of the initial conditions. The obtained state estimate $\hat{x}(t)$ is used in resolving the other task - 
optimal management of the type $\hat{u}(t)=-L(t) \hat{x}(t)$. As mentioned above, the optimal management task is usually set on the basis of the Bellman principle or the maximum principle [6]. Different quality functional, as well as the vector of functional may be used as a criterion.

\section{Concluding remarks}

Based on the use of state-of-the-art methods, models, information and innovation technologies in order to forecast the state of the non-linear dynamics of ecoeconomic and socio-humanitarian systems, integrated stochastic models of objects and processes were developed and studied, suitable for the conditions of systemic crises. The paper examines the aspects of integration of multiple business areas and sectors of the modern complex systems of SEEH type functioning and developing under the complex conditions of instability and crises. Complex formalization resulted in obtaining one of the options of the integral socio-eco-economic and innovative non-linear model of dynamics in the form of a system of differential and functional stochastic equations. The paper presents the task of forecasting the state of a complex system in the innovative economy on the basis of the integral model in a phased space with observation equations, development of optimal management filters and algorithms. The prospects of the further investigations include conducting computer experiments.

The paper deals with the problem of sustainable development and innovative integral modeling approach in the management of technogenic objects and processes as a system of socio-eco-economic and humanitarian type. Based on the use of information and innovation technologies in order to forecast the nonlinear dynamics of eco-economic and sociohumanitarian systems, integrated stochastic models of objects and processes were developed and studied, suitable for the conditions of systemic crises. The paper handles the aspect of integration of 4 business and functioning areas of the modern complex systems. It proposes a general conceptual integrated model, generalized synergetic model of dynamics, considering different uncertainty (stochastic and chaotic components). Most of the previously created dynamics models of eco-economic and socio-humanitarian systems and processes functioning and developing under the complex conditions of non-linearity, instability and crises, have theoretical and deterministic nature and are rather problematic from the viewpoint of the availability of information and adequacy for their implementation. In this connection, the objective of TOP management conditions the objective necessity of improving methods, models and information technologies on the basis of stochastic equations for SEEH management.

Integral socio-eco-economic stochastic models of the dynamics of TOP systems, i.e. technogenic integrated systems functioning under the conditions of the current systemic crises, were developed and investigated.

Based on the use of state-of-the-art methods, models, information and innovation technologies in order to forecast the state of the non-linear dynamics of eco-economic and socio-humanitarian systems, integrated stochastic models of objects and processes were developed and studied, suitable for the conditions of systemic crises. The paper approaches the aspect of integration of multiple business areas and sectors of the modern complex systems of SEEH type functioning and developing under the complex conditions of instability and crises. Complex formalization resulted in obtaining one of the options of the integral socioeco-economic and innovative non-linear model of dynamics in the form of a system of differential and functional stochastic equations.

An integrated stochastic non-linear growth dynamics model was developed and studied to forecast the development of the state of an innovative economy, which is suitable for the conditions of risks and crises. The paper examines the aspects of integration of multiple business areas and sectors of the modern complex systems functioning and developing under the present conditions of instability. The prospects for the further investigations include representation of the proposed integral model in a phased space with observation equations, development of optimal management filters and algorithms.

The prospects of the further investigations in this area include conducting computer experiments and their wide practical use. The prospects also include development and investigation of models, criteria and methods of optimal management of technogenic objects and creation of decision-making systems based on the proposed integral model within the state space.

\section{References}

1. Ramazanov, S.K., Sergienko, A.V.: Integral socioecological and economic stochastic model of dynamics of technogenic regional enterprise in the conditions of crisis. In: Solovyov, V.N. (ed.), pp. 89-108. Cherkasy (2015)

2. Ramazanov, S.K., Rogoza N.E., Musayeva E.K.: Nonlinear models and analysis of complex systems: on-tutorial manual. In: Ramazanov, S.K. (ed). Luhansk-Poltava (2009)

3. Ramazanov, S.K.: Tools of ecological and economic management of the enterprise. Donetsk (2008)

4. Ramazanov, S.K.: Modeling of social-ecologicaleconomic dynamics in an unstable environment. In: Information Sciences and Systems Science (ISN2015), pp. 284-287. Poltava (2015)

5. Ramazanov, S.K., Burbelo, O.A., Vitlinsky, V.V. et al.: Risks, security, crises and sustainable development in the economy: methodologies, models, methods of management and decisionmaking. In: Ramazanov, S.K. (ed). Luhansk (2012)

6. Ostrem, K.: Introduction to the stochastic theory of control. Moscow (1970) 
7. Grigorkiv, V.S.: Simulation of a Multisectoral Ecological-Economic System. Vol. 3, pp. 147-157. (1999)

8. Solovyov, V.I.: Economic-mathematical modeling of the software market. Moscow (2009)

9. Geets, V.M., Seminozhenko, V.P.: Innovative Prospects of Ukraine. Kharkiv (2006)

10. Klebanova, T.S., Kizim, N.A.: Models of estimation, analysis and forecasting of socioeconomic systems: monograph. Kharkiv (2010)

11. Lyashenko, I.M., Korobova, M.V.: Fundamentals of mathematical modeling of economic, ecological and social processes: taught. Ternopil (2006)

12. Ayvazyan, S.A., Afanasev, M.Yu., Rudenko, V.A.: Some questions of the specification of three-factor models of the company's production potential, taking into account intellectual capital. Vol. 3(27), pp. 78-87 (2012)

13. Kleiner, G.B.: System Economics and SystemOriented Modeling. Vol. 3, pp. 71-93 (2013)

14. Leont'ev, V., Ford, D.: Inter-industry analysis of the impact of the structure of the economy on the environment. Vol. 8, No. 3, pp. 370-400 (1972)

15. Tsukui, J., Murakami, Y.: Turnpike's Optimality in the input-output system-theory and application for planning. Amsterdam (1979)

16. Brock, W.A., Hsieh, D.: Nonlinear Dynamics, Chaos and Instability (1991)

17. Tahvonen, O., Kuuluvainen, J.: Economic growth, pollution, and renewable resources. J. Env. Econ. M. 24, 78-88 (1993)

18. Vagapova, Ya.Ya.: Modeling of economic growth taking into account ecological and social factors. Moscow (2007)

19. Moiseyev, A.N.: Optimal and balanced trajectories in models of economic growth with an endogenous form of NTP, pp. 178-190 (2004)

20. Makarov, V.L.: Computable Model of the Knowledge Economy. Vol. 45, 1, pp. 70-82 (2009)

21. Moiseev, N.N.: Mathematical problems of system analysis. Moscow (1981)

22. Kolemaev, V.A.: Economic-mathematical modeling. Modeling of macroeconomic processes and systems. Moscow (2005)

23. Krass, M.S., Chuprinov, B.P.: Mathematical methods and models for masters of economics. Saint Petersburg (2006)

24. Vorontsovsky, A.V., Vyunenko, L.E.: Forecasting the development of the economy on the basis of a hundred-hasty model of economic growth, taking into account the turning point. Vol. 5, 4, pp. 4-32. Saint Petersburg (2016)

25. Solou, R.: Theory of Growth. Panorama of the Economic Thought of the End of the 20th Century. In: Greenway, D., Blini, M., Stewart, I. (eds.). Vol. 1, pp. 479-506 (2002) 